\title{
Positive solutions for a class of sublinear elliptic systems
}

Ruyun Ma*, Ruipeng Chen and Yanqiong Lu

\section{"Correspondence:}

mary@nwnu.edu.cn

Department of Mathematics,

Northwest Normal University

Lanzhou, 730070, PR China

\section{Abstract}

In this paper, we are concerned with the existence of positive solutions of the semilinear elliptic system

$$
\begin{cases}-\Delta u_{1}=\lambda\left(g_{11}\left(u_{1}\right)+g_{12}\left(u_{2}\right)+\cdots+g_{1 n}\left(u_{n}\right)\right), & x \in \Omega, \\ -\Delta u_{2}=\lambda\left(g_{21}\left(u_{1}\right)+g_{22}\left(u_{2}\right)+\cdots+g_{2 n}\left(u_{n}\right)\right), & x \in \Omega, \\ \cdots & \\ -\Delta u_{n}=\lambda\left(g_{n 1}\left(u_{1}\right)+g_{n 2}\left(u_{2}\right)+\cdots+g_{n n}\left(u_{n}\right)\right), & x \in \Omega, \\ u_{1}(x)=u_{2}(x)=\cdots=u_{n}(x)=0, & x \in \partial \Omega,\end{cases}
$$

where $\lambda>0$ is a parameter, $g_{i j}:[0, \infty) \rightarrow[0, \infty)$ is a continuous real function for each $i, j=1,2, \ldots, n$. Under some appropriate assumptions, we show that the above system has at least one positive solution in certain interval of $\lambda$. The proofs of our main results are based upon bifurcation theory.

MSC: $34 \mathrm{~B} 15 ; 34 \mathrm{~B} 18$

Keywords: sublinear elliptic systems; positive solutions; eigenvalues; bifurcation theory

\section{Introduction}

Let $\Omega$ be a bounded smooth domain in $\mathbb{R}^{N}(N \geq 1)$. In this paper, we study the existence of positive solutions of the semilinear elliptic system

$$
\begin{cases}-\Delta u_{1}=\lambda\left(g_{11}\left(u_{1}\right)+g_{12}\left(u_{2}\right)+\cdots+g_{1 n}\left(u_{n}\right)\right), & x \in \Omega, \\ -\Delta u_{2}=\lambda\left(g_{21}\left(u_{1}\right)+g_{22}\left(u_{2}\right)+\cdots+g_{2 n}\left(u_{n}\right)\right), & x \in \Omega, \\ \cdots & \\ -\Delta u_{n}=\lambda\left(g_{n 1}\left(u_{1}\right)+g_{n 2}\left(u_{2}\right)+\cdots+g_{n n}\left(u_{n}\right)\right), & x \in \Omega, \\ u_{1}(x)=u_{2}(x)=\cdots=u_{n}(x)=0, & x \in \partial \Omega,\end{cases}
$$

where $\lambda>0$ is a bifurcation parameter, $g_{i j}:[0, \infty) \rightarrow[0, \infty)$ is a continuous real function for each $i, j=1,2, \ldots, n$.

A solution of $(1.1)$ is a pair $(\lambda, U):=\left(\lambda,\left(u_{1}, u_{2}, \ldots, u_{n}\right)\right) \in(0, \infty) \times[C(\bar{\Omega})]^{n} .(\lambda, U)$ is called a positive solution of (1.1) if $u_{i}>0$ in $\Omega$ for each $i=1,2, \ldots, n$. In the following, $\left(u_{1}, u_{2}, \ldots, u_{n}\right)$ also denotes the elements of $\mathbb{R}_{+}^{n}=\left\{\left(u_{1}, u_{2}, \ldots, u_{n}\right) \in \mathbb{R}^{n}: u_{i} \geq 0, i=\right.$ $1,2, \ldots, n\}$.

The following definitions will be used in the statement of our main results. 
Definition 1.1 [1] Let $f_{i}(i=1,2, \ldots, n)$ be smooth real functions defined on $\mathbb{R}_{+}^{n}$. Define the Jacobian of the vector field $\left(f_{1}, f_{2}, \ldots, f_{n}\right)$ as

$$
H\left(u_{1}, u_{2}, \ldots, u_{n}\right)=\left(\begin{array}{ccc}
\frac{\partial f_{1}}{\partial u_{1}} & \cdots & \frac{\partial f_{1}}{\partial u_{n}} \\
\vdots & \ddots & \vdots \\
\frac{\partial f_{n}}{\partial u_{1}} & \cdots & \frac{\partial f_{n}}{\partial u_{n}}
\end{array}\right)=\left(\begin{array}{ccc}
f_{11}^{\prime} & \cdots & f_{1 n}^{\prime} \\
\vdots & \ddots & \vdots \\
f_{n 1}^{\prime} & \cdots & f_{n n}^{\prime}
\end{array}\right) .
$$

If $\frac{\partial f_{i}}{\partial u_{j}} \geq 0(i \neq j)$ for all $\left(u_{1}, u_{2}, \ldots, u_{n}\right) \in \mathbb{R}_{+}^{n}$, then the semilinear elliptic system

$$
\begin{cases}-\Delta u_{1}=f_{1}\left(u_{1}, u_{2}, \ldots, u_{n}\right), & x \in \Omega, \\ -\Delta u_{2}=f_{2}\left(u_{1}, u_{2}, \ldots, u_{n}\right), & x \in \Omega, \\ \cdots & \\ -\Delta u_{n}=f_{n}\left(u_{1}, u_{2}, \ldots, u_{n}\right), & x \in \Omega, \\ u_{1}(x)=\cdots=u_{n}(x)=0, & x \in \partial \Omega\end{cases}
$$

is said to be cooperative. Similarly, $H$ is called a cooperative matrix.

Definition 1.2 [2] An $n \times n$ matrix $A$ is reducible if for some permutation matrix $Q$,

$$
Q A Q^{T}=\left(\begin{array}{ll}
B & 0 \\
C & D
\end{array}\right)
$$

where $B$ and $D$ are square matrices, and $Q^{T}$ is the transpose of $Q$. Otherwise, $A$ is irreducible.

In the past few years, the existence of positive solutions to sublinear semilinear elliptic systems with two equations have been extensively studied, see for example, [3-6] and the references therein. The sublinear condition plays an important role. Very recently, Wu and Cui [1] considered the existence, uniqueness and stability of positive solutions to the sublinear elliptic system (1.1). By using bifurcation theory and the continuation method, they proved the following.

\section{Theorem A Assume that}

(H1) Each $g_{i j}(i, j=1,2, \ldots, n)$ is a smooth real function defined on $\mathbb{R}_{+}$satisfying

$$
g_{i j}(0) \geq 0 \text {. }
$$

(H2) $g_{i j}^{\prime}(s) \geq 0,\left(g_{i j}(s) / s\right)^{\prime} \leq 0$ for all $s \geq 0$.

(H3) $\lim _{s \rightarrow \infty} \frac{g_{i j}(s)}{s}=0$.

(i) If at least one of $g_{i j}(0)(i=1,2, \ldots, n)$ is positive and matrix $G=\left(g_{i j}(0)\right)_{n \times n}$ is irreducible, then (1.1) has a unique positive solution $U(\lambda)=\left(u_{1}(\lambda), u_{2}(\lambda), \ldots, u_{n}(\lambda)\right)$ for all $\lambda>0$;

(ii) If $g_{i j}(0)=0, g_{i j}^{\prime}(0)>0$ for each $i, j=1,2, \ldots, n$ and matrix $G^{\prime}=\left(g_{i j}^{\prime}(0)\right)_{n \times n}$ is irreducible, then for some $\lambda_{*}>0$, (1.1) has no positive solution when $\lambda \leq \lambda_{*}$, and (1.1) has a unique positive solution $U(\lambda)=\left(u_{1}(\lambda), u_{2}(\lambda), \ldots, u_{n}(\lambda)\right)$ for $\lambda>\lambda_{*}$.

Moreover, $\left\{\left(\lambda, u_{1}(\lambda), u_{2}(\lambda), \ldots, u_{n}(\lambda)\right): \lambda>\lambda_{*}\right\}$ (in the first case, we assume $\lambda_{*}=0$ ) is a smooth curve so that $u_{i}(\lambda)$ is strictly increasing in $\lambda$, and $u_{i}(\lambda) \rightarrow 0$ as $\lambda \rightarrow \lambda_{*}^{+}$. 
We are interested in the existence of positive solutions of (1.1) under weaker assumptions. More concretely, we consider the existence of positive solutions of (1.1) from the following two aspects: (a) To obtain the counterpart of Theorem A(ii) under the weaker assumptions than those of [1]. In other words, we will not assume that $g_{i j}(i, j=1,2, \ldots, n)$ are smooth functions any more. (b) Furthermore, we will also consider the case that $\lim _{s \rightarrow 0} \frac{g_{i j}(s)}{s}(i, j=1,2, \ldots, n)$ may not exist. More precisely, the following two theorems, which are the main results of the present paper, shall be proved.

Theorem 1.1 Suppose that

(A1) $g_{i j}:[0, \infty) \rightarrow[0, \infty)(i, j=1,2, \ldots, n)$ are continuous real functions satisfying

$$
g_{i j}(0)=0 ; \quad g_{i j}(s)>0, \quad s>0 .
$$

(A2) There exist constants $g_{i j}^{0} \in(0, \infty)$ such that

$$
g_{i j}^{0}=\lim _{s \rightarrow 0} \frac{g_{i j}(s)}{s}, \quad \forall i, j=1,2, \ldots, n .
$$

(A3) $\lim _{s \rightarrow \infty} \frac{g_{i j}(s)}{s}=0, \forall i, j=1,2, \ldots, n$.

If the matrix $\left(g_{i j}^{0}\right)_{n \times n}$ is irreducible, then there exists $\hat{\lambda}>0$ such that (1.1) has no positive solution for $\lambda<\hat{\lambda}$ and (1.1) has at least one positive solution for $\lambda \geq \hat{\lambda}$.

Theorem 1.2 Let (A1) and (A3) hold. Assume the following.

(A2)' For each $i, j \in\{1,2, \ldots, n\}$, there exist $\underline{g}_{i j} \bar{g}_{i j} \in(0, \infty)$ such that

$$
\underline{g}_{i j}=\liminf _{s \rightarrow 0} \frac{g_{i j}(s)}{s} \leq \limsup _{s \rightarrow 0} \frac{g_{i j}(s)}{s}=\bar{g}_{i j} .
$$

(A4) The matrix $J_{\alpha}:=\left((1-\alpha) \underline{g}_{i j}+\alpha \bar{g}_{i j}\right)_{n \times n}$ is irreducible, where $\alpha \in[0,1]$.

Then for some $\tilde{\lambda}>0,(1.1)$ has at least one positive solution for $\lambda>\tilde{\lambda}$.

Remark 1.1 It follows from (A2) and (A2) that the matrices $\left(g_{i j}^{0}\right)_{n \times n}$ and $J_{\alpha}, \alpha \in[0,1]$ are all cooperative.

Remark 1.2 We note that our assumptions in Theorems 1.1 and 1.2 are weaker than those of Theorem A, and, accordingly, our results are weaker than Theorem A. Since we just suppose that $g_{i j}$ is continuous, we can only obtain the continua of positive solutions of (1.1) by applying bifurcation techniques, which are not necessarily curves of positive solutions, and thus the uniqueness and stability of positive solutions are not investigated. In [1], the authors obtained a smooth curve consisting of positive solutions of (1.1) by assuming stronger assumptions, under which the uniqueness and stability of positive solutions can be achieved.

Remark 1.3 For related results, established via bifurcation techniques, for other kind of problems, we refer the readers to [7-9] and the references therein. 
The rest of the paper is arranged as follows. In Section 2, we recall some basic knowledges on the maximum principle of cooperative systems as well as the eigenvalues of cooperative matrices. Finally in Section 3, we prove our main results Theorems 1.1 and 1.2 by applying bifurcation theory.

\section{Preliminaries}

We shall essentially work in Banach space $X=\left[C_{0}(\bar{\Omega})\right]^{n}$, here

$$
C_{0}(\bar{\Omega})=\{u \in C(\bar{\Omega}): u(x)=0, x \in \partial \Omega\}
$$

The norm of $U \in X$ will be defined as $\|U\|_{X}=\sum_{l=1}^{n}\left\|u_{l}\right\|$, where $\|\cdot\|$ denotes the norm of $C(\bar{\Omega})$. We use $W^{2, p}(\Omega)$ and $W_{\text {loc }}^{2, p}(\Omega)$ for the standard Sobolev space. We use $N(L)$ and $R(L)$ to denote the null and the range space of a linear operator $L$, respectively.

Let $(\lambda, U)=\left(\lambda,\left(u_{1}, u_{2}, \ldots, u_{n}\right)\right)$ be a solution of $(1.1)$. Suppose that $g_{i j}:[0, \infty) \rightarrow[0, \infty)$ $(i, j=1,2, \ldots, n)$ are smooth real functions. Then we can deduce the eigenvalue problem

$$
\begin{cases}\Delta \xi_{1}+\lambda g_{11}^{\prime} \xi_{1}+\lambda g_{12}^{\prime} \xi_{2}+\cdots+\lambda g_{1 n}^{\prime} \xi_{n}=-\mu \xi_{1}, & x \in \Omega, \\ \Delta \xi_{2}+\lambda g_{21}^{\prime} \xi_{1}+\lambda g_{22}^{\prime} \xi_{2}+\cdots+\lambda g_{2 n}^{\prime} \xi_{n}=-\mu \xi_{2}, & x \in \Omega, \\ \cdots & \\ \Delta \xi_{n}+\lambda g_{n 1}^{\prime} \xi_{1}+\lambda g_{n 2}^{\prime} \xi_{2}+\cdots+\lambda g_{n n}^{\prime} \xi_{n}=-\mu \xi_{n}, & x \in \Omega, \\ \xi_{1}(x)=\xi_{2}(x)=\cdots=\xi_{n}(x)=0, & x \in \partial \Omega,\end{cases}
$$

which can be rewritten as

$$
L \mathbf{u}=H \mathbf{u}+\mu \mathbf{u}
$$

where

$$
\mathbf{u}=\left(\begin{array}{c}
\xi_{1} \\
\vdots \\
\xi_{n}
\end{array}\right), \quad L \mathbf{u}=\left(\begin{array}{c}
-\Delta \xi_{1} \\
\vdots \\
-\Delta \xi_{n}
\end{array}\right), \quad H=\lambda\left(\begin{array}{ccc}
g_{11}^{\prime} & \cdots & g_{1 n}^{\prime} \\
\vdots & \ddots & \vdots \\
g_{n 1}^{\prime} & \cdots & g_{n n}^{\prime}
\end{array}\right)
$$

Lemma $2.1[1,4]$ Let $Y=\left[W_{\text {loc }}^{2, p}(\Omega) \cap C_{0}(\bar{\Omega})\right]^{n}$ and $Z=\left[L^{p}(\Omega)\right]^{n}$ with $p>N$. Suppose that $L, H$ are given as in (2.3), and $H$ is cooperative and irreducible. Then we have the following:

(a) $\mu_{1}=\inf \{\operatorname{Re}(\mu): \mu \in \operatorname{spt}(L-H)\}$ is a real eigenvalue of $L-H$, where $\operatorname{spt}(L-H)$ is the spectrum of $L-H$.

(b) For $\mu=\mu_{1}$, there exists a unique (up a constant multiple) eigenfunction $\mathbf{u}_{1} \in Y$, and $\mathbf{u}_{1}>0$ in $\Omega$.

(c) For $\mu<\mu_{1}$, the equation $L \mathbf{u}=H \mathbf{u}+\mu \mathbf{u}+\mathbf{f}$ is uniquely solvable for any $\mathbf{f} \in Z$, and $\mathbf{u}>0$ as long as $\mathbf{f} \geq 0$.

(d) (Maximum principle) For $\mu<\mu_{1}$, assume that $\mathbf{u} \in\left[W_{\text {loc }}^{2, p}(\Omega) \cap C(\bar{\Omega})\right]^{n}$ satisfies $L \mathbf{u} \geq H \mathbf{u}+\mu \mathbf{u}$ in $\Omega, \mathbf{u} \geq 0$ on $\partial \Omega$, then $\mathbf{u} \geq 0$ in $\Omega$.

(e) If there exists $\mathbf{u} \in\left[W_{\mathrm{loc}}^{2, p}(\Omega) \cap C(\bar{\Omega})\right]^{n}$ satisfying $L \mathbf{u} \geq H \mathbf{u}$ in $\Omega$, $\mathbf{u} \geq 0$ on $\partial \Omega$, and either $\mathbf{u} \not \equiv 0$ on $\partial \Omega$ or $L \mathbf{u} \not \equiv H \mathbf{u}$ in $\Omega$, then $\mu_{1}>0$.

For the results and proofs, see Proposition 3.1 and Theorem 1.1 of Sweers [10]. Moreover, from a standard compactness argument, there are countably many eigenvalues $\left\{\mu_{i}\right\}$ of 
$L-H$, and $\left|\mu_{i}-\mu_{1}\right| \rightarrow \infty$ as $i \rightarrow \infty$. We notice that $\mu_{i}(i \geq 2)$ are not necessarily realvalued.

In this section, we also need to consider the adjoint operator of $L-H$. Let the transpose matrix of $H$ be

$$
H^{T}=\left(\begin{array}{ccc}
g_{11}^{\prime} & \cdots & g_{n 1}^{\prime} \\
\vdots & \ddots & \vdots \\
g_{1 n}^{\prime} & \cdots & g_{n n}^{\prime}
\end{array}\right) .
$$

Then it is clear that the results in Lemma 2.1 are also true for the eigenvalue problem

$$
L \mathbf{u}^{*}=H^{T} \mathbf{u}^{*}+\mu \mathbf{u}^{*}
$$

which is equivalent to

$$
\begin{cases}\Delta \xi_{1}^{*}+\lambda g_{11}^{\prime} \xi_{1}^{*}+\lambda g_{21}^{\prime} \xi_{2}^{*}+\cdots+\lambda g_{n 1}^{\prime} \xi_{n}^{*}=-\mu \xi_{1}^{*}, & x \in \Omega, \\ \Delta \xi_{2}^{*}+\lambda g_{12}^{\prime} \xi_{1}^{*}+\lambda g_{22}^{\prime} \xi_{2}^{*}+\cdots+\lambda g_{n 2}^{\prime} \xi_{n}^{*}=-\mu \xi_{2}^{*}, & x \in \Omega, \\ \cdots & \\ \Delta \xi_{n}^{*}+\lambda g_{1 n}^{\prime} \xi_{1}^{*}+\lambda g_{2 n}^{\prime} \xi_{2}^{*}+\cdots+\lambda g_{n n}^{\prime} \xi_{n}^{*}=-\mu \xi_{n}^{*}, & x \in \Omega, \\ \xi_{1}^{*}(x)=\xi_{2}^{*}(x)=\cdots=\xi_{n}^{*}(x)=0, & x \in \partial \Omega,\end{cases}
$$

where $\mathbf{u}^{*}=\left(\xi_{1}^{*}, \xi_{2}^{*}, \ldots, \xi_{n}^{*}\right)^{T}$. It is easy to verify that $L-H^{T}$ is the adjoint operator of $L-H$, while both are considered as operators defined on subspaces of $\left[L^{2}(\Omega)\right]^{n}$.

The following lemmas are crucial in the proof of our main results.

Lemma 2.2 [1] Let $Y, Z, L$ and $H$ be the same as in Lemma 2.1. Then the principal eigenvalue $\mu_{1}$ of $L-H$ is also a real eigenvalue of $L-H^{T}, \mu_{1}=\inf \left\{\mu \in \operatorname{spt}\left(L-H^{T}\right)\right\}$, and for $\mu=\mu_{1}$, there exists a unique eigenfunction $\mathbf{u}_{1}^{*} \in\left[W_{\mathrm{loc}}^{2,2}(\Omega) \cap C_{0}(\bar{\Omega})\right]^{n}$ of $L-H^{T}$ (up a constant multiple), and $\mathbf{u}_{1}^{*}>0$ in $\Omega$.

Lemma 2.3 [11, Theorem 5.3.1] Let $n \times n$ matrix $A$ be a nonnegative irreducible matrix. Then $\rho(A)$ is a simple eigenvalue of $A$, associated to a positive eigenvector, where $\rho(A)$ denotes the spectral radius of $A$. Moreover, $\rho(A)>0$.

Lemma 2.4 [12] Let $V$ be a real Banach space. Suppose that

$$
F: \mathbb{R} \times V \rightarrow V
$$

is completely continuous and $F(\lambda, 0)=0$ for all $\lambda \in \mathbb{R}$. Let $a, b \in \mathbb{R}(a<b)$ such that $u=0$ is the isolated solution of the equation

$$
u-F(\lambda, u)=0, \quad u \in V .
$$

Furthermore, assume that

$$
d\left(I-F(a, \cdot), B_{r}(0), 0\right) \neq d\left(I-F(b, \cdot), B_{r}(0), 0\right),
$$


where $B_{r}(0)$ is an isolated neighborhood of trivial solutions. Let

$$
\mathcal{S}=\overline{\{(\lambda, u):(\lambda, u) \text { is a solution of }(2.5) \text { and } u \neq 0\}} \cup([a, b] \times\{0\}) .
$$

Then there exists a continuum (i.e., a closed connected set) $\mathcal{C}$ of $\mathcal{S}$ containing $[a, b] \times\{0\}$, and either

(i) $\mathcal{C}$ is unbounded in $\mathbb{R} \times V$; or

(ii) $\mathcal{C} \cap[(\mathbb{R} \backslash[a, b]) \times\{0\}] \neq \emptyset$.

Finally, let $\left(\lambda_{1}, \varphi_{1}\right)$ be the principal eigen-pair of the linear eigenvalue problem

$$
\begin{cases}-\Delta \varphi=\lambda \varphi, & x \in \Omega, \\ \varphi=0, & x \in \partial \Omega,\end{cases}
$$

such that $\varphi_{1}>0$ in $\Omega$ and $\left\|\varphi_{1}\right\|=1$.

\section{Proof of the main results}

Proof of Theorem 1.1. We extend each $g_{i j}$ to be a nonnegative continuous function, which is still denoted by $g_{i j}$, defined on $\mathbb{R}$ in the following way: if $s<0$, then $g_{i j}(s) \equiv g_{i j}(0)$.

Let us define

$$
F(\lambda, U)=\left(\begin{array}{c}
\Delta u_{1}+\lambda\left(g_{11}\left(u_{1}\right)+g_{12}\left(u_{2}\right)+\cdots+g_{1 n}\left(u_{n}\right)\right) \\
\Delta u_{2}+\lambda\left(g_{21}\left(u_{1}\right)+g_{22}\left(u_{2}\right)+\cdots+g_{2 n}\left(u_{n}\right)\right) \\
\cdots \\
\Delta u_{n}+\lambda\left(g_{n 1}\left(u_{1}\right)+g_{n 2}\left(u_{2}\right)+\cdots+g_{n n}\left(u_{n}\right)\right)
\end{array}\right),
$$

where $\lambda \in \mathbb{R}$. Then it follows from (A1) that $F: \mathbb{R} \times X \rightarrow X$ is continuous, and $(\lambda, U)=$ $(\lambda,(0,0, \ldots, 0))$ is always a solution of $(1.1)$. Moreover, (A2) implies that $F$ is differentiable at $(\lambda, U)=(\lambda,(0,0, \ldots, 0))$, and

$$
\begin{aligned}
F_{U}(\lambda,(0,0, \ldots, 0))\left(\begin{array}{c}
\phi_{1} \\
\phi_{2} \\
\vdots \\
\phi_{n}
\end{array}\right) & =\left(\begin{array}{c}
\Delta \phi_{1}+\lambda\left(g_{11}^{0} \phi_{1}+g_{12}^{0} \phi_{2}+\cdots+g_{1 n}^{0} \phi_{n}\right) \\
\Delta \phi_{2}+\lambda\left(g_{21}^{0} \phi_{1}+g_{22}^{0} \phi_{2}+\cdots+g_{2 n}^{0} \phi_{n}\right) \\
\cdots \\
\cdots \phi_{n}+\lambda\left(g_{n 1}^{0} \phi_{1}+g_{n 2}^{0} \phi_{2}+\cdots+g_{n n}^{0} \phi_{n}\right)
\end{array}\right) \\
& =\left(\begin{array}{c}
\Delta \phi_{1} \\
\Delta \phi_{2} \\
\vdots \\
\Delta \phi_{n}
\end{array}\right)+\lambda\left(\begin{array}{cccc}
g_{11}^{0} & g_{12}^{0} & \cdots & g_{1 n}^{0} \\
g_{21}^{0} & g_{22}^{0} & \cdots & g_{2 n}^{0} \\
\vdots & \vdots & \ddots & \vdots \\
g_{n 1}^{0} & g_{n 2}^{0} & \cdots & g_{n n}^{0}
\end{array}\right)\left(\begin{array}{c}
\phi_{1} \\
\phi_{2} \\
\vdots \\
\phi_{n}
\end{array}\right) \\
& =\left(\begin{array}{c}
\Delta \phi_{1} \\
\Delta \phi_{2} \\
\vdots \\
\Delta \phi_{n}
\end{array}\right)+\lambda J\left(\begin{array}{c}
\phi_{1} \\
\phi_{2} \\
\vdots \\
\phi_{n}
\end{array}\right),
\end{aligned}
$$

where $J=\left(g_{i j}^{0}\right)_{n \times n}$. By (A2), all entries of $J$ are positive. Therefore Lemma 2.3 yields the result that $J$ has a positive principal eigenvalue $\chi_{J}$, the corresponding eigenvector 
$\left(k_{1}, k_{2}, \ldots, k_{n}\right)^{T}$ satisfying $k_{i}>0(i=1,2, \ldots, n)$. Moreover, it is not difficult to verify that

$$
\left(\begin{array}{c}
-\Delta\left(k_{1} \varphi_{1}\right) \\
-\Delta\left(k_{2} \varphi_{1}\right) \\
\vdots \\
-\Delta\left(k_{n} \varphi_{1}\right)
\end{array}\right)=\lambda_{*} J\left(\begin{array}{c}
k_{1} \varphi_{1} \\
k_{2} \varphi_{1} \\
\vdots \\
k_{n} \varphi_{1}
\end{array}\right)
$$

where $\lambda_{*}=\frac{\lambda_{1}}{\chi_{J}}$. This implies that $\left(k_{1} \varphi_{1}, k_{2} \varphi_{1}, \ldots, k_{n} \varphi_{1}\right)^{T}$ is a positive eigenvector of the operator $F_{U}\left(\lambda_{*},(0,0, \ldots, 0)\right)$. Similarly, $J^{T}$ has the same principal eigenvalue $\chi_{J}$ and the corresponding eigenvector is $\left(k_{1}^{*}, \ldots, k_{n}^{*}\right)^{T}$, where $k_{i}^{*}(i=1,2, \ldots, n)$ is a positive constant. Obviously,

$$
\left(\begin{array}{c}
-\Delta\left(k_{1}^{*} \varphi_{1}\right) \\
-\Delta\left(k_{2}^{*} \varphi_{1}\right) \\
\vdots \\
-\Delta\left(k_{n}^{*} \varphi_{1}\right)
\end{array}\right)=\lambda_{*} J^{T}\left(\begin{array}{c}
k_{1}^{*} \varphi_{1} \\
k_{2}^{*} \varphi_{1} \\
\vdots \\
k_{n}^{*} \varphi_{1}
\end{array}\right)
$$

Hence when $\lambda=\lambda_{*}=\frac{\lambda_{1}}{\chi_{I}}, F_{U}\left(\lambda_{*},(0,0, \ldots, 0)\right)$ is not invertible and $\lambda=\lambda_{*}$ is a potential bifurcation point. More precisely, the null space

$$
N\left(F_{U}\left(\lambda_{*},(0,0, \ldots, 0)\right)\right)=\operatorname{span}\left\{\left(k_{1} \varphi_{1}, k_{2} \varphi_{1}, \ldots, k_{n} \varphi_{1}\right)^{T}\right\}
$$

is one dimensional. In addition, it is easy to see that $F_{\lambda}(\lambda, U)$ and $F_{\lambda U}(\lambda,(0,0, \ldots, 0))$ exist for $(\lambda, U) \in \mathbb{R} \times X$.

We divide the rest of the proof into two steps.

Step 1 . We show that $\left(\lambda_{*},(0,0, \ldots, 0)\right)$ is actually a bifurcation point.

Indeed, the proof of this is similar to the proof of Theorem A(ii), we state it here for the readers' convenience.

Suppose $\left(h_{1}, h_{2}, \ldots, h_{n}\right)^{T} \in R\left(F_{U}\left(\lambda_{*},(0,0, \ldots, 0)\right)\right)$. Then there exists $\left(\psi_{1}, \psi_{2}, \ldots, \psi_{n}\right) \in X$ such that

$$
F_{U}\left(\lambda_{*},(0,0, \ldots, 0)\right)\left(\begin{array}{c}
\psi_{1} \\
\psi_{2} \\
\vdots \\
\psi_{n}
\end{array}\right)=\left(\begin{array}{c}
\Delta \psi_{1} \\
\Delta \psi_{2} \\
\vdots \\
\Delta \psi_{n}
\end{array}\right)+\lambda_{*} J\left(\begin{array}{c}
\psi_{1} \\
\psi_{2} \\
\vdots \\
\psi_{n}
\end{array}\right)=\left(\begin{array}{c}
h_{1} \\
h_{2} \\
\vdots \\
h_{n}
\end{array}\right)
$$

Let us consider the adjoint eigenvalue equation

$$
\left(\begin{array}{c}
\Delta w_{1}^{*} \\
\vdots \\
\Delta w_{n}^{*}
\end{array}\right)+\lambda_{*} J^{T}\left(\begin{array}{c}
w_{1}^{*} \\
\vdots \\
w_{n}^{*}
\end{array}\right)=\left(\begin{array}{c}
\Delta w_{1}^{*} \\
\vdots \\
\Delta w_{n}^{*}
\end{array}\right)+\lambda_{*}\left(\begin{array}{ccc}
g_{11}^{0} & \cdots & g_{n 1}^{0} \\
\vdots & \ddots & \vdots \\
g_{1 n}^{0} & \cdots & g_{n n}^{0}
\end{array}\right)\left(\begin{array}{c}
w_{1}^{*} \\
\vdots \\
w_{n}^{*}
\end{array}\right)=0 \text {, }
$$

where $w_{i}^{*}=k_{i}^{*} \varphi_{1}, i=1,2, \ldots, n$. Multiplying the system (3.4) by $\left(w_{1}^{*}, w_{2}^{*}, \ldots, w_{n}^{*}\right)^{T}$, multiplying the system (3.5) by $\left(\psi_{1}, \psi_{2}, \ldots, \psi_{n}\right)^{T}$, integrating on $\Omega$ and subtracting, then we obtain

$$
\int_{\Omega} \sum_{i=1}^{n} h_{i} w_{i}^{*} d x=\int_{\Omega} \sum_{i=1}^{n} k_{i}^{*} h_{i} \varphi_{1} d x=0 .
$$


Thus $\left(h_{1}, h_{2}, \ldots, h_{n}\right)^{T} \in R\left(F_{U}\left(\lambda_{*},(0,0, \ldots, 0)\right)\right)$ if and only if (3.6) holds, which implies that $R\left(F_{U}\left(\lambda_{*},(0,0, \ldots, 0)\right)\right)$ is one dimensional.

Next, we verify that

$$
F_{\lambda U}\left(\lambda_{*},(0,0, \ldots, 0)\right)\left(\begin{array}{c}
k_{1} \varphi_{1} \\
\vdots \\
k_{n} \varphi_{1}
\end{array}\right) \notin R\left(F_{U}\left(\lambda_{*},(0,0, \ldots, 0)\right)\right) .
$$

Otherwise, we have

$$
F_{\lambda U}\left(\lambda_{*},(0,0, \ldots, 0)\right)\left(\begin{array}{c}
k_{1} \varphi_{1} \\
\vdots \\
k_{n} \varphi_{1}
\end{array}\right) \in R\left(F_{U}\left(\lambda_{*},(0,0, \ldots, 0)\right)\right)
$$

Since

$$
F_{\lambda U}\left(\lambda_{*},(0,0, \ldots, 0)\right)\left(\begin{array}{c}
k_{1} \varphi_{1} \\
\vdots \\
k_{n} \varphi_{1}
\end{array}\right)=J\left(\begin{array}{c}
k_{1} \\
\vdots \\
k_{n}
\end{array}\right) \varphi_{1}=\chi_{J}\left(\begin{array}{c}
k_{1} \\
\vdots \\
k_{n}
\end{array}\right) \varphi_{1}
$$

multiplying the system (3.9) by $\left(k_{1}^{*} \varphi_{1}, k_{2}^{*} \varphi_{1}, \ldots, k_{n}^{*} \varphi_{1}\right)^{T}$ and using (3.8), we can get a contradiction that

$$
0=\chi_{J} \cdot \int_{\Omega}\left(k_{1} k_{1}^{*}+\cdots+k_{n} k_{n}^{*}\right) \varphi_{1}^{2} d x>0
$$

By using [13, Theorem 1.7], we conclude that $\left(\lambda_{*},(0,0, \ldots, 0)\right)$ is a bifurcation point. Furthermore, by the Rabinowitz global bifurcation theorem [14], there exists a continuum $\mathcal{C}_{1}^{+}$ of positive solutions of $(1.1)$, which joins $\left(\lambda_{*},(0,0, \ldots, 0)\right)$ to infinity in $\mathbb{R} \times X$. Clearly,

$$
\mathcal{C}_{1}^{+} \cap(\{0\} \times X)=\emptyset,
$$

since (1.1) has only the trivial solution $(0,(0,0, \ldots, 0))$ when $\lambda=0$.

Step 2: We claim that $\mathcal{C}_{1}^{+}$cannot blow up at some finite $\lambda^{*} \in(0, \infty)$.

Otherwise, a sequence $\left\{\left(\lambda^{k}, U^{k}\right)\right\} \subset \mathcal{C}_{1}^{+}$can be taken such that

$$
\lim _{k \rightarrow \infty} \lambda^{k}=\lambda^{*}, \quad \lim _{k \rightarrow \infty}\left\|U^{k}\right\|_{X}=\infty
$$

where $U^{k}=\left(u_{1}^{k}, u_{2}^{k}, \ldots, u_{n}^{k}\right)$. Let $K: C_{0}(\bar{\Omega}) \rightarrow C_{0}(\bar{\Omega})$ be the Green operator of $-\Delta$ subject to Dirichlet boundary conditions, i.e., $u=K v$ if and only if

$$
\begin{cases}-\Delta u=v, & x \in \Omega \\ u(x)=0, & x \in \partial \Omega\end{cases}
$$


By the elliptic regularity, $\left(\lambda^{k}, U^{k}\right)$ satisfies

$$
\begin{cases}u_{1}^{k}(x)=\lambda^{k} K\left[g_{11}\left(u_{1}^{k}(x)\right)+g_{12}\left(u_{2}^{k}(x)\right)+\cdots+g_{1 n}\left(u_{n}^{k}(x)\right)\right], & x \in \Omega, \\ u_{2}^{k}(x)=\lambda^{k} K\left[g_{21}\left(u_{1}^{k}(x)\right)+g_{22}\left(u_{2}^{k}(x)\right)+\cdots+g_{2 n}\left(u_{n}^{k}(x)\right)\right], & x \in \Omega, \\ \cdots & \\ u_{n}^{k}(x)=\lambda^{k} k\left[g_{n 1}\left(u_{1}^{k}(x)\right)+g_{n 2}\left(u_{2}^{k}(x)\right)+\cdots+g_{n n}\left(u_{n}^{k}(x)\right)\right], & x \in \Omega .\end{cases}
$$

Here $g_{i j}$ also denotes the Nemytski operator generated by itself. Clearly, (3.12) is equivalent to

$$
\left(\begin{array}{c}
u_{1}^{k} \\
u_{2}^{k} \\
\vdots \\
u_{n}^{k}
\end{array}\right)=\lambda^{k} \mathcal{K}\left(\begin{array}{c}
g_{11}\left(u_{1}^{k}\right)+g_{12}\left(u_{2}^{k}\right)+\cdots+g_{1 n}\left(u_{n}^{k}\right) \\
g_{21}\left(u_{1}^{k}\right)+g_{22}\left(u_{2}^{k}\right)+\cdots+g_{2 n}\left(u_{n}^{k}\right) \\
\vdots \\
g_{n 1}\left(u_{1}^{k}\right)+g_{n 2}\left(u_{2}^{k}\right)+\cdots+g_{n n}\left(u_{n}^{k}\right)
\end{array}\right)
$$

where $\mathcal{K}=\operatorname{diag}(K, K, \ldots, K)$. It is well known that $K: C_{0}(\bar{\Omega}) \rightarrow C_{0}(\bar{\Omega})$ is continuous and compact, and so $\mathcal{K}$ is continuous and compact on $(0, \infty) \times X$.

Let $w_{i}^{k}=\frac{u_{i}^{k}}{\left\|u^{k}\right\|_{X}}(i=1,2, \ldots, n)$. Then $w_{i}^{k}>0$ in $\Omega$ and $\left\|\left(w_{1}^{k}, w_{2}^{k}, \ldots, w_{n}^{k}\right)\right\|_{X}=1$. Dividing both sides of (3.13) with $\left\|U^{k}\right\|_{X}$, we have

$$
\left(\begin{array}{c}
w_{1}^{k} \\
w_{2}^{k} \\
\vdots \\
w_{n}^{k}
\end{array}\right)=\lambda^{k} \mathcal{K}\left(\begin{array}{c}
\frac{g_{11}\left(u_{1}^{k}\right)}{\left\|U^{k}\right\|_{X}}+\frac{g_{12}\left(u_{2}^{k}\right)}{\left\|U^{k}\right\|_{X}}+\cdots+\frac{g_{1 n}\left(u_{n}^{k}\right)}{\left\|U^{k}\right\|_{X}} \\
\frac{g_{21}\left(u_{1}^{k}\right)}{\left\|U^{k}\right\|_{X}}+\frac{g_{22}\left(u_{2}^{k}\right)}{\left\|U^{k}\right\|_{X}}+\cdots+\frac{\left.g_{2 n} u_{n}^{k}\right)}{\left\|U^{k}\right\|_{X}} \\
\vdots \\
\frac{g_{n 1}\left(u_{1}^{k}\right)}{\left\|U^{k}\right\|_{X}}+\frac{g_{n 2}\left(u_{2}^{k}\right)}{\left\|U^{k}\right\|_{X}}+\cdots+\frac{g_{n n}\left(u_{n}^{k}\right)}{\left\|U^{k}\right\|_{X}}
\end{array}\right) .
$$

For each $i, j=1,2, \ldots, n$, from (A2) and (A3) it follows that $\frac{g_{i j}(s)}{s}$ is bounded in $[0, \infty)$. Moreover, we have

$$
\frac{g_{i j}\left(u_{j}^{k}\right)}{\left\|U^{k}\right\|_{X}} \leq \frac{g_{i j}\left(u_{j}^{k}\right)}{\left\|u_{j}^{k}\right\|}=\frac{g_{i j}\left(u_{j}^{k}\right)}{u_{j}^{k}} \cdot \frac{u_{j}^{k}}{\left\|u_{j}^{k}\right\|}
$$

Therefore,

$$
\left(\begin{array}{c}
\frac{g_{11}\left(u_{1}^{k}\right)}{\left\|U^{k}\right\|_{X}}+\frac{g_{12}\left(u_{2}^{k}\right)}{\left\|U^{k}\right\|_{X}}+\cdots+\frac{g_{1 n}\left(u_{n}^{k}\right)}{\left\|u^{k}\right\|_{X}} \\
\frac{g_{21}\left(u_{1}^{k}\right)}{\left\|U^{k}\right\|_{X}}+\frac{g_{22}\left(u_{2}^{k}\right)}{\left\|U^{k}\right\|_{X}}+\cdots+\frac{g_{2 n}\left(u_{n}^{k}\right)}{\left\|u^{k}\right\|_{X}} \\
\vdots \\
\frac{g_{n 1}\left(u_{1}^{k}\right)}{\left\|U^{k}\right\|_{X}}+\frac{g_{n 2}\left(u_{2}^{k}\right)}{\left\|U^{k}\right\|_{X}}+\cdots+\frac{g_{n n}\left(u_{n}^{k}\right)}{\left\|U^{k}\right\|_{X}}
\end{array}\right)
$$

is bounded in $X$. This together with the compactness of $\mathcal{K}$ implies that $\left\{\left(w_{1}^{k}, w_{2}^{k}, \ldots, w_{n}^{k}\right)\right\}$ has a subsequence, denoted by itself, satisfying, in $X$,

$$
\left(w_{1}^{k}, w_{2}^{k}, \ldots, w_{n}^{k}\right) \rightarrow\left(\tilde{w}_{1}, \tilde{w}_{2}, \ldots, \tilde{w}_{n}\right), \quad k \rightarrow \infty .
$$


Obviously, $\tilde{w}_{j} \geq 0(j=1,2, \ldots, n)$ in $\Omega$ and $\left\|\left(\tilde{w}_{1}, \tilde{w}_{2}, \ldots, \tilde{w}_{n}\right)\right\|_{X}=1$. In addition, we have $\lambda^{*}>0$. Or else, let $k \rightarrow \infty$, then by (3.14) we get $\tilde{w}_{j} \equiv 0(j=1,2, \ldots, n)$ in $\Omega$, which contradicts $\left\|\left(\tilde{w}_{1}, \tilde{w}_{2}, \ldots, \tilde{w}_{n}\right)\right\|_{X}=1$.

We define

$$
\Omega_{j}^{+}=\left\{x \in \Omega: \tilde{w}_{j}(x)>0\right\}, \quad \Omega_{j}^{0}=\Omega \backslash \Omega_{j}^{+}, \quad j=1,2, \ldots, n .
$$

Then for each $j=1,2, \ldots, n$,

$$
u_{j}^{k}(x)=w_{j}^{k}(x) \cdot\left\|U^{k}\right\|_{X} \rightarrow+\infty \quad \text { as } k \rightarrow \infty, x \in \Omega_{j}^{+},
$$

by Lebesgue control convergence theorem, we get

$$
\frac{g_{i j}\left(u_{j}^{k}(x)\right)}{u_{j}^{k}(x)} \rightarrow 0 \quad \text { as } k \rightarrow \infty, x \in \Omega_{j}^{+}, \forall i, j=1,2, \ldots, n,
$$

which together with (3.15) yields

$$
\frac{g_{i j}\left(u_{j}^{k}(x)\right)}{\left\|U^{k}\right\|_{X}} \rightarrow 0 \quad \text { as } k \rightarrow \infty, x \in \Omega_{j}^{+}, \forall i, j=1,2, \ldots, n .
$$

On the other hand, we know from (A2) and (3.15) that

$$
\frac{g_{i j}\left(u_{j}^{k}(x)\right)}{\left\|U^{k}\right\|_{X}} \rightarrow 0 \quad \text { as } k \rightarrow \infty, x \in \Omega_{j}^{0}, \forall i, j=1,2, \ldots, n .
$$

Hence we conclude from (3.17) and (3.18) that

$$
\frac{g_{i j}\left(u_{j}^{k}(x)\right)}{\left\|U^{k}\right\|_{X}} \rightarrow 0 \quad \text { as } k \rightarrow \infty, x \in \Omega, \forall i, j=1,2, \ldots, n .
$$

Now, let $k \rightarrow \infty$ in (3.14), using (3.19) and the fact that $\lambda^{*}>0$ we can obtain

$$
\tilde{w}_{j}(x)=0 \quad \text { in } \Omega, \text { for all } j=1,2, \ldots, n,
$$

which contradicts $\left\|\left(\tilde{w}_{1}, \tilde{w}_{2}, \ldots, \tilde{w}_{n}\right)\right\|_{X}=1$.

Finally, by (3.10), the connectness of $\mathcal{C}_{1}^{+}$and above arguments, we can find some $\hat{\lambda}>0$ such that (1.1) has no positive solution for $\lambda<\hat{\lambda}$, and (1.1) has at least one positive solution for $\lambda \geq \hat{\lambda}$.

To prove Theorem 1.2, we need the following lemmas as required.

By Remark 1.1 and Lemma 2.3, the matrices $J_{0}=\left(g_{i j}\right)_{n \times n}$ and $J_{1}=\left(\bar{g}_{i j}\right)_{n \times n}$ have the principal eigenvalues $\chi_{0}:=\rho\left(J_{0}\right)>0$ and $\chi_{1}:=\rho\left(J_{1}\right)>0$, respectively, and the corresponding positive eigenvectors are $\left(k_{1}^{0}, k_{2}^{0}, \ldots, k_{n}^{0}\right)^{T}$ and $\left(k_{1}^{1}, k_{2}^{1}, \ldots, k_{n}^{1}\right)^{T}$. Moreover, it is easy to obtain

$$
\left(\begin{array}{c}
\Delta\left(k_{1}^{0} \varphi_{1}\right) \\
\Delta\left(k_{2}^{0} \varphi_{1}\right) \\
\vdots \\
\Delta\left(k_{n}^{0} \varphi_{1}\right)
\end{array}\right)+\frac{\lambda_{1}}{\chi_{0}}\left(\begin{array}{cccc}
g_{11} & \underline{g}_{12} & \cdots & \underline{g}_{1 n} \\
\underline{g}_{21} & \underline{g}_{22} & \cdots & \underline{g}_{2 n} \\
\vdots & \vdots & \ddots & \vdots \\
\underline{g}_{n 1} & \underline{g}_{n 2} & \cdots & \underline{g}_{n n}
\end{array}\right)\left(\begin{array}{c}
k_{1}^{0} \varphi_{1} \\
k_{2}^{0} \varphi_{1} \\
\vdots \\
k_{n}^{0} \varphi_{1}
\end{array}\right)=0
$$


and

$$
\left(\begin{array}{c}
\Delta\left(k_{1}^{1} \varphi_{1}\right) \\
\Delta\left(k_{2}^{1} \varphi_{1}\right) \\
\vdots \\
\Delta\left(k_{n}^{1} \varphi_{1}\right)
\end{array}\right)+\frac{\lambda_{1}}{\chi_{1}}\left(\begin{array}{cccc}
\bar{g}_{11} & \bar{g}_{12} & \cdots & \bar{g}_{1 n} \\
\bar{g}_{21} & \bar{g}_{22} & \cdots & \bar{g}_{2 n} \\
\vdots & \vdots & \ddots & \vdots \\
\bar{g}_{n 1} & \bar{g}_{n 2} & \cdots & \bar{g}_{n n}
\end{array}\right)\left(\begin{array}{c}
k_{1}^{1} \varphi_{1} \\
k_{2}^{1} \varphi_{1} \\
\vdots \\
k_{n}^{1} \varphi_{1}
\end{array}\right)=0
$$

where $\lambda_{1}$ is given as in (2.6). By Lemma 2.2, the matrices $\left(J_{0}\right)^{T}$ and $\left(J_{1}\right)^{T}$ have principal eigenvalues $\chi_{0}$ and $\chi_{1}$, respectively, the associated positive eigenvectors are $\left(k_{1}^{0 *}, k_{2}^{0 *}\right.$, $\left.\ldots, k_{n}^{0 *}\right)^{T}$ and $\left(k_{1}^{1 *}, k_{2}^{1 *}, \ldots, k_{n}^{1 *}\right)^{T}$. We can easily verify that

$$
\left(\begin{array}{c}
\Delta\left(k_{1}^{0 *} \varphi_{1}\right) \\
\Delta\left(k_{2}^{0 *} \varphi_{1}\right) \\
\vdots \\
\Delta\left(k_{n}^{0 *} \varphi_{1}\right)
\end{array}\right)+\frac{\lambda_{1}}{\chi_{0}}\left(\begin{array}{lllc}
\underline{g}_{11} & \underline{g}_{21} & \cdots & \underline{g}_{n 1} \\
\underline{g}_{12} & \underline{g}_{22} & \cdots & \underline{g}_{n 2} \\
\vdots & \vdots & \ddots & \vdots \\
\underline{g}_{1 n} & \underline{g}_{2 n} & \cdots & \underline{g}_{n n}
\end{array}\right)\left(\begin{array}{c}
k_{1}^{0 *} \varphi_{1} \\
k_{2}^{0 *} \varphi_{1} \\
\vdots \\
k_{n}^{0 *} \varphi_{1}
\end{array}\right)=0
$$

and

$$
\left(\begin{array}{c}
\Delta\left(k_{1}^{1 *} \varphi_{1}\right) \\
\Delta\left(k_{2}^{1 *} \varphi_{1}\right) \\
\vdots \\
\Delta\left(k_{n}^{1 *} \varphi_{1}\right)
\end{array}\right)+\frac{\lambda_{1}}{\chi_{1}}\left(\begin{array}{cccc}
\bar{g}_{11} & \bar{g}_{21} & \cdots & \bar{g}_{n 1} \\
\bar{g}_{12} & \bar{g}_{22} & \cdots & \bar{g}_{n 2} \\
\vdots & \vdots & \ddots & \vdots \\
\bar{g}_{1 n} & \bar{g}_{2 n} & \cdots & \bar{g}_{n n}
\end{array}\right)\left(\begin{array}{c}
k_{1}^{1 *} \varphi_{1} \\
k_{2}^{1 *} \varphi_{1} \\
\vdots \\
k_{n}^{1 *} \varphi_{1}
\end{array}\right)=0 .
$$

Let $\Sigma \subset(0, \infty) \times X$ be the closure of the set of positive solutions to (1.1). We extend each $g_{i j}$ to be a function defined on $\mathbb{R}$ by

$$
\tilde{g}_{i j}(s)= \begin{cases}g_{i j}(s), & s \in[0, \infty), \\ g_{i j}(0), & s \in(-\infty, 0),\end{cases}
$$

then $\tilde{g}_{i j}(s) \geq 0$ on $\mathbb{R}$. Let $(\lambda, U) \in(0, \infty) \times X$ be a solution of

$$
\begin{cases}-\Delta u_{1}=\lambda\left(\tilde{g}_{11}\left(u_{1}\right)+\tilde{g}_{12}\left(u_{2}\right)+\cdots+\tilde{g}_{1 n}\left(u_{n}\right)\right), & x \in \Omega, \\ -\Delta u_{2}=\lambda\left(\tilde{g}_{21}\left(u_{1}\right)+\tilde{g}_{22}\left(u_{2}\right)+\cdots+\tilde{g}_{2 n}\left(u_{n}\right)\right), & x \in \Omega, \\ \cdots & \\ -\Delta u_{n}=\lambda\left(\tilde{g}_{n 1}\left(u_{1}\right)+\tilde{g}_{n 2}\left(u_{2}\right)+\cdots+\tilde{g}_{n n}\left(u_{n}\right)\right), & x \in \Omega, \\ u_{1}(x)=u_{2}(x)=\cdots=u_{n}(x)=0, & x \in \partial \Omega .\end{cases}
$$

Then by (3.24), for each $i=1,2, \ldots, n$,

$$
u_{i}(x)=\lambda K\left[\tilde{g}_{i 1}\left(u_{1}(x)\right)+\tilde{g}_{i 2}\left(u_{2}(x)\right)+\cdots+\tilde{g}_{i n}\left(u_{n}(x)\right)\right] \geq 0, \quad x \in \Omega,
$$

where $K$ is given as in the proof of Theorem 1.1. Hence $(\lambda, U)$ is a nonnegative solution of (3.25). Moreover, from (3.24) it follows that $(\lambda, U)$ is a solution of (1.1). On the other hand, (3.25) has no half-trivial solutions. Otherwise, $U$ must have trivial and nontrivial components, and so there is a $i_{0} \in\{1,2, \ldots, n\}$ such that $u_{i_{0}} \equiv 0$ in $\Omega$, and by the maximum 
principle of elliptic boundary value problems, we have

$$
0=u_{i_{0}}=\lambda K\left[\tilde{g}_{i_{0} 1}\left(u_{1}\right)+\tilde{g}_{i_{0} 2}\left(u_{2}\right)+\cdots+\tilde{g}_{i_{0} n}\left(u_{n}\right)\right]>0, \quad x \in \Omega,
$$

which is a contradiction. Therefore, the closure of the set of nontrivial solutions of (3.25) is exactly $\Sigma$.

In the following, we shall apply the Leray-Schauder degree theory, mainly to the mapping $\Phi_{\lambda}: X \rightarrow X$,

$$
\Phi_{\lambda}(U)=U-\lambda \mathcal{K} g(U)
$$

where $\mathcal{K}$ is given as in (3.13), and

$$
g(U)(x)=\left(\begin{array}{c}
\tilde{g}_{11}\left(u_{1}\right)(x)+\tilde{g}_{12}\left(u_{2}\right)(x)+\cdots+\tilde{g}_{1 n}\left(u_{n}\right)(x) \\
\tilde{g}_{21}\left(u_{1}\right)(x)+\tilde{g}_{22}\left(u_{2}\right)(x)+\cdots+\tilde{g}_{2 n}\left(u_{n}\right)(x) \\
\vdots \\
\tilde{g}_{n 1}\left(u_{1}\right)(x)+\tilde{g}_{n 2}\left(u_{2}\right)(x)+\cdots+\tilde{g}_{n n}\left(u_{n}\right)(x)
\end{array}\right), \quad x \in \Omega
$$

is the associated Nemytski operator. For $R>0$, let $B_{R}=\left\{U \in X:\|U\|_{X}<R\right\}$, let $d\left(\Phi_{\lambda}, B_{R}, 0\right)$ denote the degree of $\Phi_{\lambda}$ on $B_{R}$ with respect to 0 .

Lemma 3.1 Let $\Lambda \subset \mathbb{R}^{+}$be a compact interval with $\Lambda \cap\left[\frac{\lambda_{1}}{\chi_{1}}, \frac{\lambda_{1}}{\chi_{0}}\right]=\emptyset$. Then there exists a $\delta_{1}>0$ such that

$$
\Phi_{\lambda}(U) \neq 0, \quad \forall U \in X, \quad 0<\|U\|_{X} \leq \delta_{1}, \quad \forall \lambda \in \Lambda .
$$

Proof Suppose on the contrary that there exist sequences $\left\{\lambda^{k}\right\} \subset \Lambda$ and $\left\{U^{k}\right\} \subset X$ so that

$$
\begin{aligned}
& \Phi_{\lambda^{k}}\left(U^{k}\right)=0, \quad \forall k \in \mathbb{N}, \\
& \lambda^{k} \rightarrow \lambda^{*} \quad \text { and } \quad\left\|U^{k}\right\|_{X} \rightarrow 0, \quad k \rightarrow \infty .
\end{aligned}
$$

Apparently, (3.27) is equivalent to

$$
\begin{cases}-\Delta u_{1}^{k}=\lambda^{k}\left(\tilde{g}_{11}\left(u_{1}^{k}\right)+\tilde{g}_{12}\left(u_{2}^{k}\right)+\cdots+\tilde{g}_{1 n}\left(u_{n}^{k}\right)\right), & x \in \Omega, \\ -\Delta u_{2}^{k}=\lambda^{k}\left(\tilde{g}_{21}\left(u_{1}^{k}\right)+\tilde{g}_{22}\left(u_{2}^{k}\right)+\cdots+\tilde{g}_{2 n}\left(u_{n}^{k}\right)\right), & x \in \Omega, \\ \cdots & \\ -\Delta u_{n}^{k}=\lambda^{k}\left(\tilde{g}_{n 1}\left(u_{1}^{k}\right)+\tilde{g}_{n 2}\left(u_{2}^{k}\right)+\cdots+\tilde{g}_{n n}\left(u_{n}^{k}\right)\right), & x \in \Omega, \\ u_{1}^{k}(x)=u_{2}^{k}(x)=\cdots=u_{n}^{k}(x)=0, & x \in \partial \Omega\end{cases}
$$

and $u_{i}^{k} \geq 0(i=1,2, \ldots, n)$ in $\Omega$, and therefore $\tilde{g}_{i j}\left(u_{j}^{k}\right)=g_{i j}\left(u_{j}^{k}\right), \forall i, j=1,2, \ldots, n$. Furthermore, it follows from (A2)' that, for $s>0$ sufficiently small, $g_{i j} s \leq g_{i j}(s) \leq \bar{g}_{i j} s$. This together with (3.28) implies that, for $k$ large enough,

$$
\underline{g}_{i j} \cdot u_{j}^{k}(s) \leq g_{i j}\left(u_{j}^{k}(s)\right) \leq \bar{g}_{i j} \cdot u_{j}^{k}(s), \quad \forall i, j=1,2, \ldots, n .
$$


Multiplying (3.29) by $\left(k_{1}^{0 *} \varphi_{1}, k_{2}^{0 *} \varphi_{1}, \ldots, k_{n}^{0 *} \varphi_{1}\right)^{T}$, multiplying (3.22) by $\left(u_{1}^{k}, u_{2}^{k}, \ldots, u_{n}^{k}\right)^{T}$, integrating on $\Omega$ and adding, using (3.30) and the fact $\tilde{g}_{i j}\left(u_{j}^{k}\right)=g_{i j}\left(u_{j}^{k}\right), \forall i, j=1,2, \ldots, n$, we know that, for $k$ large enough,

$$
\begin{aligned}
\frac{\lambda_{1}}{\chi_{0}} \int_{\Omega} \sum_{i=1}^{n}\left[\sum_{j=1}^{n} \underline{g}_{i j} k_{i}^{0 *} u_{j}^{k} \varphi_{1}\right] d x & =\lambda^{k} \int_{\Omega} \sum_{i=1}^{n}\left[\sum_{j=1}^{n} g_{i j}\left(u_{j}^{k}\right) k_{i}^{0 *} \varphi_{1}\right] d x \\
& \geq \lambda^{k} \int_{\Omega} \sum_{i=1}^{n}\left[\sum_{j=1}^{n} \underline{g}_{i j} k_{i}^{0 *} u_{j}^{k} \varphi_{1}\right] d x,
\end{aligned}
$$

and so $\lambda^{k} \leq \frac{\lambda_{1}}{\chi_{0}}$ for $k$ sufficiently large. Similarly, by (3.23) and (3.30), we can deduce that $\lambda^{k} \geq \frac{\lambda_{1}}{\chi_{1}}$ for $k$ large enough. Consequently, for $k$ sufficiently large we get $\lambda^{k} \in\left[\frac{\lambda_{1}}{\chi_{1}}, \frac{\lambda_{1}}{\chi_{0}}\right]$, which contradicts $\left\{\lambda^{k}\right\} \subset \Lambda$.

Corollary 3.2 For $\lambda \in\left(0, \frac{\lambda_{1}}{\chi_{1}}\right)$ and $\delta \in\left(0, \delta_{1}\right), d\left(\Phi_{\lambda}, B_{\delta}, 0\right)=1$.

Proof Lemma 3.1, applied to the interval $\Lambda=[0, \lambda]$, guarantees the existence of $\delta_{1}>0$ such that, for $\delta \in\left(0, \delta_{1}\right)$,

$$
U-\tau \lambda \mathcal{K} g(U) \neq 0, \quad U \in X, 0<\|U\|_{X} \leq \delta, \tau \in[0,1] .
$$

Hence for any $\delta \in\left(0, \delta_{1}\right)$,

$$
d\left(\Phi_{\lambda}, B_{\delta}, 0\right)=d\left(I, B_{\delta}, 0\right)=1
$$

Lemma 3.3 Suppose that $\lambda>\frac{\lambda_{1}}{\chi_{0}}$. Then there exists $\delta_{2}>0$ such that

$$
\Phi_{\lambda}(U) \neq \tau \varphi, \quad \forall U \in X: 0<\|U\|_{X} \leq \delta_{2}, \forall \tau \geq 0
$$

where $\varphi=\left(k_{1}^{0} \varphi_{1}, k_{2}^{0} \varphi_{1}, \ldots, k_{n}^{0} \varphi_{1}\right)^{T}$.

Proof Suppose on the contrary that there exist $\tau_{k} \geq 0$ and a sequence $\left\{U^{k}\right\}$ with $\left\|U^{k}\right\|_{X}>0$ and $\left\|U^{k}\right\|_{X} \rightarrow 0$ such that

$$
\Phi_{\lambda}\left(U^{k}\right)=\tau_{k} \varphi, \quad \forall k \in \mathbb{N},
$$

which is

$$
\begin{cases}-\Delta u_{1}^{k}=\lambda\left(g_{11}\left(u_{1}^{k}\right)+g_{12}\left(u_{2}^{k}\right)+\cdots+g_{1 n}\left(u_{n}^{k}\right)\right)+\lambda_{1} \tau_{k} k_{1}^{0} \varphi_{1}, & x \in \Omega, \\ -\Delta u_{2}^{k}=\lambda\left(g_{21}\left(u_{1}^{k}\right)+g_{22}\left(u_{2}^{k}\right)+\cdots+g_{2 n}\left(u_{n}^{k}\right)\right)+\lambda_{1} \tau_{k} k_{2}^{0} \varphi_{1}, & x \in \Omega, \\ \cdots & \\ -\Delta u_{n}^{k}=\lambda\left(g_{n 1}\left(u_{1}^{k}\right)+g_{n 2}\left(u_{2}^{k}\right)+\cdots+g_{n n}\left(u_{n}^{k}\right)\right)+\lambda_{1} \tau_{k} k_{n}^{0} \varphi_{1}, & x \in \Omega, \\ u_{1}^{k}(x)=u_{2}^{k}(x)=\cdots=u_{n}^{k}(x)=0, & x \in \partial \Omega .\end{cases}
$$

Clearly, $u_{i}^{k} \geq 0(i=1,2, \ldots, n)$ in $\Omega$. Multiplying (3.22) by $\left(u_{1}^{k}, u_{2}^{k}, \ldots, u_{n}^{k}\right)^{T}$, multiplying (3.32) by $\varphi^{*}:=\left(k_{1}^{0 *} \varphi_{1}, k_{2}^{0 *} \varphi_{1}, \ldots, k_{n}^{0 *} \varphi_{1}\right)^{T}$, integrating over $\Omega$ and adding, then by (3.30) we 
know that, for $k$ large enough,

$$
\begin{aligned}
\frac{\lambda_{1}}{\chi_{0}} \int_{\Omega} \sum_{i=1}^{n}\left[\sum_{j=1}^{n} \underline{g}_{i j} k_{i}^{0 *} u_{j}^{k} \varphi_{1}\right] d x & =\lambda \int_{\Omega} \sum_{i=1}^{n}\left[\sum_{j=1}^{n} g_{i j}\left(u_{j}^{k}\right) k_{i}^{0 *} \varphi_{1}\right] d x+\lambda_{1} \tau_{k} \sum_{i=1}^{n} k_{i}^{0} k_{i}^{0 *} \varphi_{1}^{2} \\
& \geq \lambda \int_{\Omega} \sum_{i=1}^{n}\left[\sum_{j=1}^{n} g_{i j}\left(u_{j}^{k}\right) k_{i}^{0 *} \varphi_{1}\right] d x \\
& \geq \lambda \int_{\Omega} \sum_{i=1}^{n}\left[\sum_{j=1}^{n} g_{i j} k_{i}^{0 *} u_{j}^{k} \varphi_{1}\right] d x .
\end{aligned}
$$

Hence $\lambda \leq \frac{\lambda_{1}}{\chi_{0}}$ for $k$ sufficiently large, which contradicts $\lambda>\frac{\lambda_{1}}{\chi_{0}}$.

Corollary 3.4 For $\lambda>\frac{\lambda_{1}}{\chi_{0}}$ and $\delta \in\left(0, \delta_{2}\right), d\left(\Phi_{\lambda}, B_{\delta}, 0\right)=0$.

Proof Let $0<\delta \leq \delta_{2}$, where $\delta_{2}$ is the constant given as in Lemma 3.3. Since $\Phi_{\lambda}$ is bounded in $\bar{B}_{\delta}$, there exists a constant $C>0$ such that $\Phi_{\lambda}(U) \neq C \varphi, \forall U \in \bar{B}_{\delta}$. By Lemma 3.3, we get

$$
\Phi_{\lambda}(U) \neq \tau C \varphi, \quad U \in \partial B_{\delta}, \tau \in[0,1] .
$$

Hence,

$$
d\left(\Phi_{\lambda}, B_{\delta}, 0\right)=d\left(\Phi_{\lambda}-C \varphi, B_{\delta}, 0\right)=0 .
$$

Proof of Theorem 1.2. For $n \in \mathbb{N}$ such that $\frac{\lambda_{1}}{\chi_{1}}-\frac{1}{n}>0$, let $a_{n}=\frac{\lambda_{1}}{\chi_{1}}-\frac{1}{n}, b_{n}=\frac{\lambda_{1}}{\chi_{0}}+\frac{1}{n}$ and $\hat{\delta}=\min \left\{\delta_{1}, \delta_{2}\right\}$. For any $\delta \in(0, \hat{\delta})$, it is easy to see that the assumptions of Lemma 2.4 are all satisfied. Therefore there exists a continuum $\mathcal{C}$ of solutions of $(3.25)$ containing $\left[a_{n}, b_{n}\right] \times$ $\{0\}$, and either

(i) $\mathcal{C}$ is unbounded in $\mathbb{R} \times X$; or

(ii) $\mathcal{C} \cap\left[\left(\mathbb{R} \backslash\left[a_{n}, b_{n}\right]\right) \times\{0\}\right] \neq \emptyset$.

By Lemma 3.1, the case (ii) cannot occur, and hence $\mathcal{C}$ is unbounded bifurcated from $\left[a_{n}, b_{n}\right] \times\{0\}$. Note that (3.25) has only trivial solutions when $\lambda=0$, and therefore $\mathcal{C} \cap(\{0\} \times$ $X)=\emptyset$. Moreover, from Lemma 3.1 it follows that for a closed interval $I \subset\left[a_{n}, b_{n}\right] \backslash\left[\frac{\lambda_{1}}{\chi_{1}}, \frac{\lambda_{1}}{\chi_{0}}\right]$, if $U \in\{U \in X:(\lambda, U) \in \Sigma, \lambda \in I\}$, then $\|U\|_{X} \rightarrow 0$ in $X$ is impossible. Thus $\mathcal{C}$ must be bifurcated from $\left[\frac{\lambda_{1}}{\chi_{1}}, \frac{\lambda_{1}}{\chi_{0}}\right]$. Finally, applying similar methods to the proof of Step 2 of Theorem 1.1, we can show that

$$
\{\lambda:(\lambda, U) \in \mathcal{C}\} \supset\left(\frac{\lambda_{1}}{\chi_{0}}, \infty\right) .
$$

Consequently, (1.1) has at least one positive solution for $\lambda>\tilde{\lambda}:=\frac{\lambda_{1}}{\chi_{0}}$.

Competing interests

The authors declare that they have no competing interests.

Authors' contributions

RM and RC completed the main study, carried out the results of this article and drafted the manuscript. YL checked the proofs and verified the calculation. All the authors read and approved the final manuscript. 


\section{Acknowledgements}

The authors are very grateful to the anonymous referees for their valuable suggestions. This work was supported by the NSFC (No. 11361054, No. 11201378), SRFDP(No. 20126203110004), Gansu provincial National Science Foundation of China (No. 1208RJZA258)

Received: 17 October 2013 Accepted: 9 January 2014 Published: 30 Jan 2014

\section{References}

1. Wu, B, Cui, R: Existence, uniqueness and stability of positive solutions to a general sublinear elliptic systems. Bound. Value Probl. 74, 1-14 (2013)

2. Serrin, J, Zou, H: Existence of positive entire solutions of elliptic Hamiltonian systems. Commun. Partial Differ. Equ. 23, 577-599 (1998)

3. Chen, Z, Chern, J, Shi, J, Tang, Y: On the uniqueness and structure of solutions to a coupled elliptic system. J. Differ. Equ. 249, 3419-3442 (2010)

4. Chern, J, Tang, Y, Lin, C, Shi, J: Existence, uniqueness and stability of positive solutions to sublinear elliptic systems. Proc. R. Soc. Edinb. A 141, 45-64 (2011)

5. Cui, R, Li, P, Shi, J, Wang, Y: Existence, uniqueness and stability of positive solutions for a class of semilinear elliptic systems. Topol. Methods Nonlinear Anal. 42, 91-104 (2013)

6. Shi, J, Shivaji, R: Exact multiplicity of positive solutions to cooperative elliptic systems. Preprint (2012)

7. Chen, R, Ma, R: Global bifurcation of positive radial solutions for an elliptic system in reactor dynamics. Comput. Math. Appl. 65(8), 1119-1128(2013)

8. Ma, R, Dai, G: Global bifurcation and nodal solutions for a Sturm-Liouville problem with a nonsmooth nonlinearity. J. Funct. Anal. 265, 1443-1459 (2013)

9. Dai, G, Ma, R: Unilateral global bifurcation phenomena and nodal solutions for p-Laplacian. J. Differ. Equ. 252, 2448-2468 (2012)

10. Sweers, G: Strong positivity in $C(\bar{\Omega})$ for elliptic systems. Math. Z. 209, 251-271 (1992)

11. Serre, D: Matrices: Theory and Applications. Graduate Texts in Mathematics, vol. 216. Springer, Berlin (2002)

12. Rabinowitz, $\mathrm{PH}$ : Some aspects of nonlinear eigenvalue problems. Rocky Mountain Consortium Symposium on Nonlinear Eigenvalue Problems (Santa Fe, N. M., 1971). Rocky Mt. J. Math. 3, 161-202 (1973)

13. Crandall, MG, Rabinowitz, PH: Bifurcation from simple eigenvalues. J. Funct. Anal. 8, 321-340 (1971)

14. Rabinowitz, PH: Some global results for nonlinear eigenvalue problems. J. Funct. Anal. 7, 487-513 (1971)

10.1186/1687-2770-2014-28

Cite this article as: Ma et al.: Positive solutions for a class of sublinear elliptic systems. Boundary Value Problems

$2014,2014: 28$

\section{Submit your manuscript to a SpringerOpen ${ }^{\ominus}$ journal and benefit from:}

- Convenient online submission

- Rigorous peer review

- Immediate publication on acceptance

- Open access: articles freely available online

- High visibility within the field

- Retaining the copyright to your article 